\title{
ASYMPTOTIC STABILITY OF SOLUTIONS FOR A KIND OF THIRD-ORDER STOCHASTIC DIFFERENTIAL EQUATIONS WITH DELAYS
}

\author{
AYMAN M. MAHMOUD AND CEMIL TUNÇ
}

Received 21 December, 2018

\begin{abstract}
This work is devoted to investigate the stochastic asymptotically stability of the zero solution for a kind of third-order stochastic differentials equation with variable and constant delays by a suitable Lyapunov functional. Our results improve and form a complement to some results that can be found in the literature. In the last section, we give an example to illustrate our main result.
\end{abstract}

2010 Mathematics Subject Classification: 34K20; 34K50; 37B25

Keywords: asymptotic stability, stochastic differential equation with delay, third-order, Lyapunov functional

\section{INTRODUCTION}

Stochastic delay differential equations (SDDEs) are natural generalizations of stochastic ordinary differential equations (SODEs) by allowing the coefficients to depend on the past values.

Recently, the studies of stochastic differential equations (SDEs) have attracted the considerable attentions of many scholars in the last forty years.

SDEs play an important role in many branches of science and engineering, and there are a large number of books, which provide full details for the background of probability theory and stochastic calculus, see for example, $[6,7,9,10,13-16]$ and the references therein.

Systems of SDDEs occupy now a place of central importance in many areas of science including medicine, engineering, biology and physics.

Stability theory is one of the main components of SDDEs. The Lyapunov's direct method has been successfully used to investigate stability problems in deterministic SDDEs for more than one hundred years, when there is no analytical expression for solutions.

An apparent advantage of this method is the stability in the large can be obtained without any prior knowledge of solutions. Therefore the method yields stability information directly without solving differential equations. 
However, there are many difficulties encountered in the study of stability by means of Lyapunov's direct method. Therefore, in the relevant literature, some authors investigated the stability of solutions for DDEs and SDDEs by using different approaches such as the fixed point method, the inequalities techniques, the perturbation methods, the second method of Lyapunov and so on (see e.g. [19,22] and reference therein).

In this direction, many authors have proposed different approaches to investigate the stability of solutions of third-order DDEs. We can mention the papers of Ademola et al. [4,5], Graef and Tunç [8], Mahmoud [11], Omeike [17], Oudjedi et al. [18], Remili et al. [20,21], Sadek [23], Shekhar et al. [24], Tunç [25-29] and the references cited therein.

Meanwhile, the scarcity of works on stability and boundedness of solutions for third-order SDEs with or without delay were studied very rarely, interesting results are contained, for instance, in [1], [2], [3].

In 2015, Abou-El-Ela et al. [1] considered the stochastic asymptotic stability of the zero solution and the uniform stochastic boundedness of all solutions for the third-order SDE of the form

$$
\dddot{x}(t)+a \ddot{x}(t)+b \dot{x}(t)+c x(t)+\sigma x(t) \dot{\omega}(t)=p(t, x(t), \dot{x}(t), \ddot{x}(t)),
$$

where $a, b, c$ and $\sigma$ are positive constants; $\omega(t) \in \mathbb{R}^{m}$ is a standard Wiener process, $p$ is a continuous function.

In 2015, Abou-El-Ela et al. [2] investigated the asymptotic stability of the zero solution for the third-order SDDEs given by

$$
\begin{aligned}
& \dddot{x}(t)+a_{1} \ddot{x}(t)+g_{1}\left(\dot{x}\left(t-r_{1}(t)\right)\right)+f_{1}(x(t))+\sigma_{1} x(t) \dot{\omega}(t)=0, \\
& \dddot{x}(t)+a_{2} \ddot{x}(t)+f_{2}(x(t)) \dot{x}(t)+f_{3}\left(x\left(t-r_{2}(t)\right)\right)+\sigma_{2} x(t-h(t)) \dot{\omega}(t)=0,
\end{aligned}
$$

where $a_{1}, a_{2}, \sigma_{1}$ and $\sigma_{2}$ are positive constants; $0 \leq r_{1}(t) \leq \gamma_{1}, 0 \leq r_{2}(t) \leq \gamma_{2}, \gamma_{1}$ and $\gamma_{2}$ are two positive constants which will be determined later. $0 \leq h(t), \sup h(t)=H$; $\omega(t) \in \mathbb{R}^{m}$ is a standard Wiener process; $g_{1}, f_{1}, f_{2}$ and $f_{3}$ are continuous functions with $g_{1}(0)=f_{1}(0)=f_{3}(0)=0$.

In 2017, Ademola [3] studied the problems of stability, boundedness and uniqueness of solutions of a certain third-order SDDE as the following form

$$
\dddot{x}(t)+a \ddot{x}(t)+b \dot{x}(t)+h(x(t-\tau))+\sigma x(t) \dot{\omega}(t)=p(t, x(t), \dot{x}(t), \ddot{x}(t)),
$$

where $a, b$ and $\sigma$ are positives constants, $h, p$ are nonlinear continuous functions in their respective arguments with $h(0)=0, \tau>0$ is a constant delay.

The main purpose of this work is to establish new criteria for the stochastic asymptotic stability of the zero solution for a kind of third-order nonlinear SDE with variable and constant delays as the following form

$$
\dddot{x}(t)+a \ddot{x}(t)+\phi(\dot{x}(t-r(t)))+\psi(x(t-r(t))+\sigma x(t-h) \dot{\omega}(t)=0,
$$


where $a, \sigma$ and $h$ are positive constants, $r(t)$ is a continuously differentiable function with $0 \leq r(t) \leq \gamma_{1}, \gamma_{1}$ is a positive constant which will be determined later, $\phi, \psi$ are two nonlinear continuous functions in their respective arguments with $\phi(0)=\psi(0)=$ $0, \omega(t)=\left(\omega_{1}(t), \omega_{2}(t), \cdots, \omega_{n}(t)\right) \in \mathbb{R}^{m}$ is $m-$ dimensional standard Brownian motion, defined on the probability space. The functions $\phi$ and $\psi$ are also differentiable throughout this work.

In this paper, by constructing a suitable Lyapunov functional, sufficient conditions for the stochastically asymptotically stability of the zero solution of (1.1) are established. Our result includes and improves the former results that can be found in the literature.

The remainder of this work is organized as follows. In section 2, we give a theorem, which deals with stochastic asymptotically stability of the zero solution for (1.1). In section 3, we introduced the proof of the main theorem. In the last section, we gave an example to verify the analysis made in this work.

\section{Stability Result}

Let $\omega(t)=\left(\omega_{1}(t), \ldots, \omega_{m}(t)\right)$ be an $m$-dimensional Brownian motion defined on the probability space. Consider an $n$-dimensional SDE

$$
d x(t)=f(t, x(t)) d t+g(t, x(t)) d B(t) \text { on } t \geq 0,
$$

with initial value $x(0)=x_{0} \in \mathbb{R}^{n}$. As a standing condition, we assume that $f$ : $\mathbb{R}^{+} \times \mathbb{R}^{n} \rightarrow \mathbb{R}^{n}$ and $g: \mathbb{R}^{+} \times \mathbb{R}^{n} \rightarrow \mathbb{R}^{n \times m}$ satisfy the local Lipschitzian condition and the linear growth condition for the existence and uniqueness of solutions for equation (2.1) (see for example, $[12,30]$ ). It is therefore known that equation $(2.1)$ has a unique continuous solution on $t \geq 0$, which is denoted by $x\left(t ; x_{0}\right)$ in this work. Assume furthermore that $f(t, 0)=0$ and $g(t, 0)=0$, for all $t \geq 0$. Hence the stochastic differential equation admits the zero solution $x(t ; 0) \equiv 0$.

Let $C^{1,2}\left(\mathbb{R}^{+} \times \mathbb{R}^{n} ; \mathbb{R}^{+}\right)$denote the family of non-negative functions $V(t, x)$ defined on $\mathbb{R}^{+} \times \mathbb{R}^{n}$, which are once continuously differentiable in $t$ and twice continuously differentiable in $x$.

Define the differential operator $\mathscr{L}$ associated with equation (2.1) by

$$
\mathscr{L}=\frac{\partial}{\partial t}+\sum_{i=1}^{n} f_{i}(t, x) \frac{\partial}{\partial x_{i}}+\frac{1}{2} \sum_{i, j=1}^{n}\left[g(t, x) g^{T}(t, x)\right]_{i j} \frac{\partial^{2}}{\partial x_{i} \partial x_{j}},
$$

If $\mathscr{L}$ acts on a function $V \in C^{1,2}\left(\mathbb{R}^{+} \times \mathbb{R}^{n} ; \mathbb{R}^{+}\right)$, then

$$
\mathscr{L} V(t, x)=V_{t}(t, x)+V_{x}(t, x) \cdot f(t, x)+\frac{1}{2} \operatorname{trace}\left[g^{T}(t, x) V_{x x}(t, x) g(t, x)\right],
$$


where $V_{t}=\frac{\partial V}{\partial t}, V_{x}=\left(\frac{\partial V}{\partial x_{1}}, \ldots, \frac{\partial V}{\partial x_{n}}\right)$ and

$$
V_{x x}=\left(\frac{\partial^{2} V}{\partial x_{i} \partial x_{j}}\right)_{n \times n}=\left(\begin{array}{ccc}
\frac{\partial^{2} V}{\partial x_{1} \partial x_{1}} & \cdots & \frac{\partial^{2} V}{\partial x_{1} \partial x_{n}} \\
\vdots & & \vdots \\
\frac{\partial^{2} V}{\partial x_{n} \partial x_{1}} & \cdots & \frac{\partial^{2} V}{\partial x_{n} \partial x_{n}}
\end{array}\right) .
$$

Moreover, let $\mathcal{K}$ denote the family of all continuous nondecreasing functions $\theta$ : $\mathbb{R}^{+} \rightarrow \mathbb{R}^{+}$such that $\theta(0)=0$ and $\theta(r)>0$, if $r>0$.

Lemma 1 ([13]). Assume that there exist $V \in C^{1,2}\left(\mathbb{R}^{+} \times \mathbb{R}^{n} ; \mathbb{R}^{+}\right)$and $\theta \in \mathcal{K}$ such that

$$
\begin{gathered}
V(t, 0)=0, \quad \theta(|x|) \leq V(t, x), \text { and } \\
\mathscr{L} V(t, x) \leq 0, \text { for all }(t, x) \in \mathbb{R}^{+} \times \mathbb{R}^{n} .
\end{gathered}
$$

Then the zero solution of the stochastic differential equation (2.1) is stochastically stable.

Lemma 2 ([13]). Assume that there exist $V \in C^{1,2}\left(\mathbb{R}^{+} \times \mathbb{R}^{n} ; \mathbb{R}^{+}\right)$and $\theta_{1}, \theta_{2}$, $\theta_{3} \in \mathcal{K}$ such that

$$
\begin{gathered}
\theta_{1}(|x|) \leq V(t, x) \leq \theta_{2}(|x|), \text { and } \\
\mathscr{L} V(t, x) \leq-\theta_{3}(|x|), \text { for all }(t, x) \in \mathbb{R}^{+} \times \mathbb{R}^{n} .
\end{gathered}
$$

Then the zero solution of the stochastic differential equation (2.1) is stochastically asymptotically stable.

Now we present the main stability result of (1.1).

Theorem 1. In additions to the basic assumptions imposed on the functions $\phi$ and $\psi$ appearing in (1.1), suppose that there exists positive constants $\alpha_{1}, \alpha_{2}, \beta_{1}, \beta_{2}, \gamma_{1}, \gamma_{2}$, $c_{1}, L$ and $M$ such that:

(i) $\alpha_{1} \leq \frac{\psi(x)}{x} \leq \alpha_{2}$ and $\psi(x) \operatorname{sgn} x>0$, for all $x \neq 0$.

(ii) $\sup \left\{\psi^{\prime}(x)\right\}=\frac{c_{1}}{2}$ and $\left|\psi^{\prime}(x)\right| \leq L$, for all $x$.

(iii) $\beta_{1} \leq \frac{\phi(y)}{y} \leq \beta_{2}$, for all $y \neq 0$ and $\left|\phi^{\prime}(y)\right| \leq M$, for all $y$.

(iv) $0 \leq r(t) \leq \gamma_{1}$ and $r^{\prime}(t) \leq \gamma_{2}$, such that $0<\gamma_{2}<1$.

(v) $a \beta_{1}-c_{1}>2 \beta_{1}+6$.

(vi) $\sigma^{2}<2 \alpha_{1}-a-\beta_{1}-2$.

Then the zero solution of (1.1) is stochastically asymptotically stable, provided that

$$
\begin{aligned}
& \gamma_{1}<\min \left\{\frac{2 \alpha_{1}-\sigma^{2}-a-\beta_{1}-2}{2(L+M)}, \frac{\left(a \beta_{1}-c_{1}-2 \beta_{1}-6\right)\left(1-\gamma_{2}\right)}{4 \lambda(L+M)\left(1-\gamma_{2}\right)+4 L(\lambda+2)},\right. \\
& \left.\frac{\left(a \beta_{1}-c_{1}-2 \beta_{1}\right)\left(1-\gamma_{2}\right)}{4 \beta_{1}(L+M)\left(1-\gamma_{2}\right)+4 \beta_{1}(\lambda+2) M}\right\} \text {, }
\end{aligned}
$$

with $\lambda=\frac{a \beta_{1}+c_{1}}{4 \beta_{1}}$. 


\section{Proof of TheOREM 1}

The equation (1.1) can be written in the following equivalent system:

$$
\begin{aligned}
\dot{x}= & y, \\
\dot{y}= & z, \\
\dot{z}= & -a z-\phi(y)-\psi(x)+\int_{t-r(t)}^{t} \phi^{\prime}(y(s)) z(s) d s+\int_{t-r(t)}^{t} \psi^{\prime}(x(s)) y(s) d s \\
& -\sigma x(t-h) \dot{\omega}(t) .
\end{aligned}
$$

Define the Lyapunov functional $V\left(t, X_{t}\right)$, where $X_{t}=\left(x_{t}, y_{t}, z_{t}\right)$, as the following

$$
\begin{aligned}
V\left(t, X_{t}\right)= & \lambda \int_{0}^{x} \psi(\xi) d \xi+\psi(x) y+\frac{1}{2} \lambda a y^{2}+\int_{0}^{y} \phi(\eta) d \eta+\lambda y z+\frac{1}{2} z^{2} \\
& +x z+x^{2}+\mu \int_{-r(t)}^{0} \int_{t+s}^{t} y^{2}(\vartheta) d \vartheta d s \\
& +v \int_{-r(t)}^{0} \int_{t+s}^{t} z^{2}(\vartheta) d \vartheta d s+\frac{1}{2} \sigma^{2} \int_{t-h}^{t} x^{2}(s) d s,
\end{aligned}
$$

where $\mu$ and $v$ are two positive constants, which will be determined later.

Our target here is to show that the Lyapunov functional $V\left(t, X_{t}\right)$ satisfies the conditions of Lemma 2.

Thus from (3.2), (3.1) and by using Itô formula (2.2), we get

$$
\begin{aligned}
\mathscr{L} V\left(t, X_{t}\right)= & \psi^{\prime}(x) y^{2}+\lambda z^{2}-\lambda \phi(y) y-a z^{2}+y z-a x z-x \phi(y)-x \psi(x)+2 x y \\
& +(x+\lambda y+z)\left(\int_{t-r(t)}^{t} \phi^{\prime}(y(s)) z(s) d s+\int_{t-r(t)}^{t} \psi^{\prime}(x(s)) y(s) d s\right) \\
& +\mu r(t) y^{2}-\mu\left(1-r^{\prime}(t)\right) \int_{t-r(t)}^{t} y^{2}(\vartheta) d \vartheta \\
& +v r(t) z^{2}-v\left(1-r^{\prime}(t)\right) \int_{t-r(t)}^{t} z^{2}(\vartheta) d \vartheta+\frac{1}{2} \sigma^{2} x^{2} .
\end{aligned}
$$

In view the assumptions $(i)-(i v)$ of Theorem 1 , we obtain

$$
\begin{aligned}
\mathscr{L} V\left(t, X_{t}\right) \leq & \frac{c_{1}}{2} y^{2}+\lambda z^{2}-\lambda \beta_{1} y^{2}-a z^{2}+y z-a x z-\beta_{1} x y-\alpha_{1} x^{2}+2 x y+\frac{1}{2} \sigma^{2} x^{2} \\
& +(x+\lambda y+z)\left(M \int_{t-r(t)}^{t} z(s) d s+L \int_{t-r(t)}^{t} y(s) d s\right) \\
& +\mu \gamma_{1} y^{2}+v \gamma_{1} z^{2}-\left(1-\gamma_{2}\right)\left(\mu \int_{t-r(t)}^{t} y^{2}(\vartheta) d \vartheta+v \int_{t-r(t)}^{t} z^{2}(\vartheta) d \vartheta\right) .
\end{aligned}
$$


Then by using the inequality $2 u v \leq u^{2}+v^{2}$, with the condition $r(t) \leq \gamma_{1}$ in Theorem 1 , we have

$$
\begin{aligned}
\mathscr{L} V\left(t, X_{t}\right) \leq & -\left\{\alpha_{1}-\frac{1}{2}\left(\sigma^{2}+a+\beta_{1}+2\right)-\frac{1}{2}(L+M) \gamma_{1}\right\} x^{2} \\
& -\left\{\lambda \beta_{1}-\frac{1}{2}\left(c_{1}+\beta_{1}+3\right)-\frac{1}{2} \lambda(L+M) \gamma_{1}-\mu \gamma_{1}\right\} y^{2} \\
& -\left\{\frac{1}{2}(a-1)-\frac{1}{2}(L+M) \gamma_{1}-\lambda-v \gamma_{1}\right\} z^{2} \\
& +\left\{L+\frac{\lambda L}{2}-\mu\left(1-\gamma_{2}\right)\right\} \int_{t-r(t)}^{t} y^{2}(\vartheta) d \vartheta \\
& +\left\{M+\frac{\lambda M}{2}-v\left(1-\gamma_{2}\right)\right\} \int_{t-r(t)}^{t} z^{2}(\vartheta) d \vartheta
\end{aligned}
$$

If we take

$$
\mu=\frac{L(\lambda+2)}{2\left(1-\gamma_{2}\right)}>0 \text { and } v=\frac{M(\lambda+2)}{2\left(1-\gamma_{2}\right)}>0,
$$

it follows that

$$
\begin{aligned}
\mathscr{L} V\left(t, X_{t}\right) \leq & -\left\{\alpha_{1}-\frac{1}{2}\left(\sigma^{2}+a+\beta_{1}+2\right)-\frac{1}{2}(L+M) \gamma_{1}\right\} x^{2} \\
& -\left\{\lambda \beta_{1}-\frac{1}{2}\left(c_{1}+\beta_{1}+3\right)-\frac{1}{2} \lambda(L+M) \gamma_{1}-\frac{L(\lambda+2)}{2\left(1-\gamma_{2}\right)} \gamma_{1}\right\} y^{2} \\
& -\left\{\frac{1}{2}(a-1)-\frac{1}{2}(L+M) \gamma_{1}-\lambda-\frac{M(\lambda+2)}{2\left(1-\gamma_{2}\right)} \gamma_{1}\right\} z^{2} .
\end{aligned}
$$

In view of

$$
\lambda \beta_{1}-\frac{1}{2} c_{1}=\frac{a \beta_{1}-c_{1}}{4}>0 \text { and } \frac{a}{2}-\lambda=\frac{a \beta_{1}-c_{1}}{4 \beta_{1}}>0,
$$

We have

$$
\begin{aligned}
\mathscr{L} V\left(t, X_{t}\right) \leq & -\left\{\alpha_{1}-\frac{1}{2}\left(\sigma^{2}+a+\beta_{1}+2\right)-\frac{L+M}{2} \gamma_{1}\right\} x^{2} \\
& -\left\{\frac{1}{4}\left(a \beta_{1}-c_{1}-2 \beta_{1}-6\right)-\frac{\lambda(L+M)\left(1-\gamma_{2}\right)+L(\lambda+2)}{2\left(1-\gamma_{2}\right)} \gamma_{1}\right\} y^{2} \\
& -\left\{\frac{a \beta_{1}-c_{1}-2 \beta_{1}}{4 \beta_{1}}-\frac{(L+M)\left(1-\gamma_{2}\right)+M(\lambda+2)}{2\left(1-\gamma_{2}\right)} \gamma_{1}\right\} z^{2} .
\end{aligned}
$$

Thus, in view of (3.3), one can conclude that $\mathscr{L} V\left(t, X_{t}\right)$ satisfies the condition (ii) of Lemma 2 as:

$$
\mathscr{L} V\left(t, X_{t}\right) \leq-D_{1}\left(x^{2}+y^{2}+z^{2}\right), \text { for some } D_{1}>0,
$$


provided that

$$
\begin{gathered}
\gamma_{1}<\min \left\{\frac{2 \alpha_{1}-\sigma^{2}-a-\beta_{1}-2}{2(L+M)}, \frac{\left(a \beta_{1}-c_{1}-2 \beta_{1}-6\right)\left(1-\gamma_{2}\right)}{4 \lambda(L+M)\left(1-\gamma_{2}\right)+4 L(\lambda+2)},\right. \\
\left.\frac{\left(a \beta_{1}-c_{1}-2 \beta_{1}\right)\left(1-\gamma_{2}\right)}{4 \beta_{1}(L+M)\left(1-\gamma_{2}\right)+4 \beta_{1}(\lambda+2) M}\right\} .
\end{gathered}
$$

Next, we shall show that the assumption $(i)$ of Lemma 2 is satisfied.

Since $\int_{-r(t)}^{0} \int_{t+s}^{t} y^{2}(\vartheta) d \vartheta d s$ and $\int_{-r(t)}^{0} \int_{t+s}^{t} z^{2}(\vartheta) d \vartheta d s$ are non-negative and by using the assumption ( i i i ) of Theorem 1, we obtain

$$
\begin{aligned}
V\left(t, X_{t}\right) \geq & \lambda \int_{0}^{x} \psi(\xi) d \xi+\psi(x) y+\frac{1}{2} \lambda a y^{2}+\frac{1}{2} \beta_{1} y^{2}+\lambda y z+\frac{1}{2} z^{2}+x z+x^{2} \\
= & \frac{1}{2 \beta_{1}}\left(\beta_{1} y+\psi(x)\right)^{2}+\left(\lambda y+\frac{z}{2}\right)^{2}+\left(x+\frac{z}{2}\right)^{2}+\frac{1}{2} \lambda(a-2 \lambda) y^{2} \\
& +\frac{2}{\beta_{1} y^{2}} \int_{0}^{x} \psi(\xi)\left\{\int_{0}^{y}\left(\lambda \beta_{1}-\psi^{\prime}(\xi)\right) \eta d \eta\right\} d \xi ; y \neq 0 .
\end{aligned}
$$

Now we recall that:

$$
a-2 \lambda=\frac{a \beta_{1}-c_{1}}{2 \beta_{1}}>0,
$$

and

$$
\left.\lambda \beta_{1}-\psi^{\prime}(\xi) \geq \frac{a \beta_{1}+c_{1}}{4}-\frac{c_{1}}{2}=\frac{a \beta_{1}-c_{1}}{4}>0 ; \text { by condition ( } i i\right) \text { of Theorem } 1 .
$$

Then, we get

$$
\begin{aligned}
\frac{2}{\beta_{1} y^{2}} & \int_{0}^{x} \psi(\xi)\left\{\int_{0}^{y}\left(\lambda \beta_{1}-\psi^{\prime}(\xi)\right) \eta d \eta\right\} d \xi \\
& \geq \frac{a \beta_{1}-c_{1}}{4 \beta_{1}} \int_{0}^{y} \psi(\xi) d \xi,
\end{aligned}
$$

which together with (3.5), implies the following inequality

$$
\begin{aligned}
V\left(t, X_{t}\right) \geq & \frac{1}{2 \beta_{1}}\left(\beta_{1} y+\psi(x)\right)^{2}+\left(x+\frac{z}{2}\right)^{2}+\frac{1}{2} \lambda\left(\frac{a \beta_{1}-c_{1}}{2 \beta_{1}}\right) y^{2} \\
& +\left(\lambda y+\frac{z}{2}\right)^{2}+\frac{a \beta_{1}-c_{1}}{4 \beta_{1}} \int_{0}^{y} \psi(\xi) d \xi .
\end{aligned}
$$

Hence, we can see that

$$
V\left(t, X_{t}\right) \geq D_{2}\left(x^{2}+y^{2}+z^{2}\right), \quad \text { for some } D_{2}>0 .
$$

In view of the assumptions $\psi(x) \leq \alpha_{2} x, \psi(y) \leq \beta_{2} y$ from the conditions $(i)$ and (iii) of Theorem 1 respectively; and the inequality $u v \leq \frac{1}{2}\left(u^{2}+v^{2}\right)$, then we can 
write from (3.2) that

$$
\begin{aligned}
V\left(t, X_{t}\right) \leq & \lambda \int_{0}^{x} \alpha_{1} \xi d \xi+\frac{\alpha_{2}}{2}\left(x^{2}+y^{2}\right)+\frac{1}{2} \lambda a y^{2}+\int_{0}^{y} \beta_{2} \eta d \eta+\frac{\lambda}{2}\left(y^{2}+z^{2}\right) \\
& +\frac{1}{2} z^{2}+\frac{1}{2}\left(x^{2}+z^{2}\right)+x^{2}+\mu \int_{t-r(t)}^{t}(\vartheta-t+r(t)) y^{2}(\vartheta) d \vartheta \\
& +v \int_{t-r(t)}^{t}(\vartheta-t+r(t)) z^{2}(\vartheta) d \vartheta+\frac{1}{2} \sigma^{2} \int_{t-h}^{t} x^{2}(s) d s .
\end{aligned}
$$

Since $r(t) \leq \gamma_{1}$, then it follows that

$$
\begin{aligned}
V\left(t, X_{t}\right) \leq & \frac{1}{2}\left\{(\lambda+1) \alpha_{2}+3+\sigma^{2} h\right\}\|x\|^{2}+\frac{1}{2}\left\{\alpha_{2}+\beta_{2}+\lambda(a+1)+\mu \gamma_{1}^{2}\right\}\|y\|^{2} \\
& +\frac{1}{2}\left\{\lambda+2+v \gamma_{1}^{2}\right\}\|z\|^{2} .
\end{aligned}
$$

Then there exists a positive constant $D_{3}$ such that

$$
V\left(t, X_{t}\right) \leq D_{3}\left(x^{2}+y^{2}+z^{2}\right), D_{3}>0 .
$$

Therefore from (3.6) and (3.7), we note that $V\left(t, X_{t}\right)$ satisfies condition $(i)$ of Lemma 2.

Thus all the assumptions of Lemma 2 are satisfied, so the zero solution of (1.1) is stochastically asymptotically stable.

This completes the proof of Theorem 1.

\section{EXAMPLE}

In this section, we give an example to show the applicability of the result obtained and for illustrations.

As an application of Theorem 1, we consider the third-order stochastic delay differential equation as the following form:

$$
\begin{aligned}
\dddot{x}(t) & +12 \ddot{x}(t)+8 \dot{x}(t-r(t))+\sin (\dot{x}(t-r(t)))+24 x(t-r(t)) \\
& +\frac{x(t-r(t))}{1+x^{2}(t-r(t))}+3 x(t) \dot{\omega}(t)=0 .
\end{aligned}
$$

Its equivalent system is given as:

$$
\begin{aligned}
\dot{x}= & y, \\
\dot{y}= & z, \\
\dot{z}= & -12 z-(8 y+\sin y)-\left(24 x+\frac{x}{1+x^{2}}\right)+\int_{t-r(t)}^{t}\{8+\cos y(s)\} z(s) d s \\
& +\int_{t-r(t)}^{t}\left\{24+\frac{1-x^{2}(s)}{\left(1+x^{2}(s)\right)^{2}}\right\} y(s) d s-2 x(t) \dot{\omega}(t) .
\end{aligned}
$$


By comparing the above differential system (3.1) and taking into account the assumptions of Theorem 1.

The path of the function siny is shown in Figure 1. It follows that

$$
a=12, \phi(y)=8 y+\sin y, \frac{\phi(y)}{y}-8=\frac{\sin y}{y},
$$

then we find

$$
-1 \leq \frac{\phi(y)}{y}-8 \leq 1
$$

Hence, we have

$$
\beta_{1}=7, \beta_{2}=9 \text {, also }\left|\phi^{\prime}(y)\right|=|8+\cos y| \leq 9=M .
$$

The behaviour of $\frac{x}{1+x^{2}}$ is shown in Figure 2. Therefore we obtain

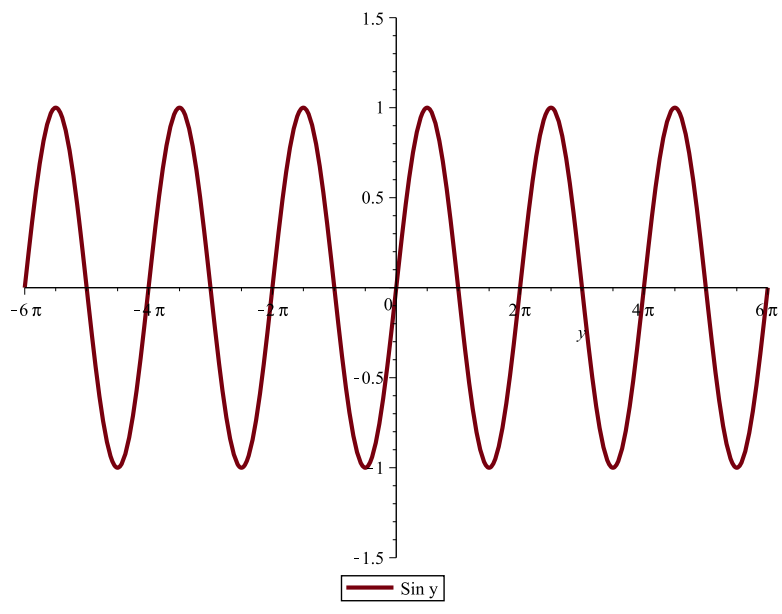

FIGURE 1. The behaviour of the function $\sin y$

$$
\psi(x)=24 x+\frac{x}{1+x^{2}}, \quad \frac{\psi(x)}{x}-24=\frac{1}{1+x^{2}}, \text { then } 0 \leq \frac{\psi(x)}{x}-24 \leq 1 .
$$

It tends to

$$
\begin{gathered}
\alpha_{1}=24, \alpha_{2}=25, \psi^{\prime}(x)=24+\frac{1-x^{2}}{\left(1+x^{2}\right)^{2}}, \text { since } \\
\left|\frac{1-x^{2}}{\left(1+x^{2}\right)^{2}}\right| \leq 1 \text {, then }\left|\psi^{\prime}(x)\right| \leq 25=L .
\end{gathered}
$$

Therefore $\sup \left\{\left|\psi^{\prime}(x)\right|\right\}=25$, then we obtain $c_{1}=50$, and $\lambda=\frac{a \beta_{1}+c_{1}}{4 \beta_{1}}=\frac{67}{14}$. It is obvious that

$$
a \beta_{1}-c_{1}=34 \text { and } 2 \beta_{1}+6=20 .
$$




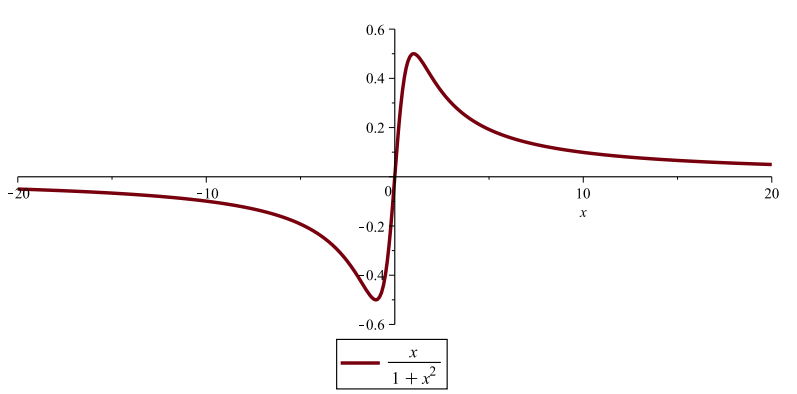

FIGURE 2. The behaviour of the function $\frac{x}{1+x^{2}}$

\section{Therefore}

$$
a \beta_{1}-c_{1}>2 \beta_{1}+6 \text {, and } 2 \alpha_{1}-a-\beta_{1}-2=27>\sigma^{2}=9 \text { (since } \sigma=3 \text { ). }
$$

Thus, the above estimates show all the assumptions of Theorem 1 hold, so we can prove that

$$
\begin{aligned}
\mathscr{L} V\left(t, X_{t}\right) \leq & \left(9-17 \gamma_{1}\right) x^{2}-\left\{\frac{14}{4}-\frac{1}{2}(163+2 \mu) \gamma_{1}\right\} y^{2} \\
& -\left\{\frac{10}{14}-(17+v) \gamma_{1}\right\} z^{2}+\left\{85-\mu\left(1-\gamma_{2}\right)\right\} \int_{t-r(t)}^{t} y^{2}(\vartheta) d \vartheta \\
& +\left\{30.5-v\left(1-\gamma_{2}\right)\right\} \int_{t-r(t)}^{t} z^{2}(\vartheta) d \vartheta .
\end{aligned}
$$

Let us choose

$$
\mu=\frac{85}{1-\gamma_{2}}>0 \text { and } v=\frac{30.5}{1-\gamma_{2}}>0
$$

where $0<\gamma_{2}<1$.

Consequently, it follows for a positive constant $\rho_{1}$ that

$$
\mathscr{L} V\left(t, X_{t}\right) \leq-\rho_{1}\left(x^{2}+y^{2}+z^{2}\right)
$$

provided that

$$
\gamma_{1}<\min \left\{\frac{9}{34}, \frac{7\left(1-\gamma_{2}\right)}{326\left(1-\gamma_{2}\right)+340}, \frac{5\left(1-\gamma_{2}\right)}{14\left\{17\left(1-\gamma_{2}\right)+30.5\right\}}\right\} .
$$

Also, we can see that

$$
\begin{aligned}
V\left(t, X_{t}\right) \geq & \frac{1}{14}(7 y+\psi(x))^{2}+\left(\frac{67}{14} y+\frac{z}{2}\right)^{2}+\left(x+\frac{z}{2}\right)^{2}+5.8 y^{2} \\
& +\frac{17}{14} \int_{0}^{y} \psi(\xi) d \xi .
\end{aligned}
$$


Then, there exists a positive constant $\rho_{2}$ such that

$$
V\left(t, X_{t}\right) \geq \rho_{2}\left(x^{2}+y^{2}+z^{2}\right) .
$$

As well it can be shown that:

$$
V\left(t, X_{t}\right) \leq \frac{157}{2}\|x\|^{2}+\frac{1}{2}\left\{96+\frac{25(\lambda+2)}{2\left(1-\gamma_{2}\right)} \gamma_{1}^{2}\right\}\|y\|^{2}+\frac{1}{2}\left\{\frac{95}{14}+\frac{9(\lambda+2)}{2\left(1-\gamma_{2}\right)} \gamma_{1}^{2}\right\}\|z\|^{2} .
$$

Hence there exists a positive constant $\rho_{3}$ satisfying

$$
V\left(t, X_{t}\right) \leq \rho_{3}\left(x^{2}+y^{2}+z^{2}\right) .
$$

Now from the results (4.2), (4.3) and (4.4), we note that all the conditions of Lemma 2 are satisfied, then the zero solution of (4.1) is stochastically asymptotically stable.

\section{ACKNOWLEDGEMENT}

The authors of this paper would like to express their sincere appreciation to the anonymous referee for his/her valuable comments and suggestions which have led to an improvement in the presentation of the paper.

\section{REFERENCES}

[1] A. M. A. Abou-El-Ela, A. I. Sadek, A. M. Mahmoud, and E. S. Farghaly, "New stability and boundedness results for solutions of a certain third-order nonlinear stochastic differential equation." Asian Journal of Mathematics and Computer Research, vol. 5, no. 1, pp. 60-70, 2015.

[2] A. M. A. Abou-El-Ela, A. I. Sadek, A. M. Mahmoud, and E. S. Farghaly, "Stability of solutions for certain third-order nonlinear stochastic delay differential equation." Ann. of Appl. Math., vol. 31, no. 3, pp. 253-261, 2015.

[3] A. T. Ademola, "Stability, boundedness and uniqueness of solutions to certain third-order stochastic delay differential equations." Differ. Equ. Control Processes, vol. 2, pp. 24-50, 2017.

[4] A. T. Ademola and P. O. Arawomo, "Uniform stability and boundedness of solutions of nonlinear delay differential equations of the third-order." Mathematical Journal Okayama University, vol. 55, pp. 157-166, 2013.

[5] A. T. Ademola, B. S. Ogundare, M. O. Ogundiran, and O. Adesina, "Stability; boundedness and existence of periodic solutions to certain third-order delay differential equations with multiple deviating arguments." International Journal of Differential Equations, vol. 2015, pp. 1-12, 2015, doi: 10.1155/2015/213935.

[6] A. Friedman, Stochastic Differential Equations and Applications. New York: Academic Press, 1976.

[7] I. I. Gikhman and A. V. Skorokhod, Stochastic Differential Equations. Berline, Heidelberg, New York: Springer-Verlag, 1972.

[8] J. R. Graef and C. Tunç, "Global asymptotic stability and boundedness of certain multi-delay functional differential equations of third-order." Math. Meth. Appl. Sci., vol. 38, no. 17, pp. 37473752, 2015, doi: 10.1002/mma.3314.

[9] R. Z. Khasminskii, Stochastic Stability of Differential Equations. Germantown: Sijthoff and Noordhoff, 1980.

[10] G. J. Kushner, Stochastic Stability and Control. Moscow: World Publ. Co., 1969. 
[11] A. M. Mahmoud, "On the asymptotic stability of solutions for a certain non-autonomous thirdorder delay differential equation." British Journal of Mathematics and Computer Science, vol. 16, no. 3, pp. 1-12, 2016, doi: 10.9734/BJMCS/2016/23936.

[12] X. Mao, "Existence and uniqueness of solutions of stochastic integral equations." J. Fuzhou Univ., vol. 4, pp. 41-50, 1983.

[13] X. Mao, Stochastic Differential Equations and Their Applications. Chichester: Horwood Publishing, 1997.

[14] X. Mao, Stochastic Differential Equations and Applications (2nd Ed.). United Kingdom: Woodhead Publishing Limited, 2007.

[15] S. E. A. Mohammed, Stochastic Functional Differential Equations. Boston, London, Melbourne: Pitman Advanced Publishing Program, 1984.

[16] B. Oksendal, Stochastic Differential Equations, An Introduction with Applications. New York: Springer-Verlag, 2000.

[17] M. O. Omeike, "New results on the stability of solution of some non-autonomous delay differential equations of the third-order." Differ. Equ. Control Processes, vol. 1, pp. 18-29, 2010.

[18] L. Oudjedi, D. Beldjerd, and M. Remili, "On the stability of solutions for non-autonomous delay differential equations of third-order." Differ. Equ. Control Processes, vol. 1, pp. 22-34, 2014

[19] S. Polidoro and M. A. Ragusa, "Hölder regularity for solutions of ultraparabolic equations in divergence form." Potential Analysis, vol. 14, no. 4, pp. 341-350, 2001, doi: 10.1023/A:1011261019736.

[20] M. Remili and D. Beldjerd, "Boundedness and stability results for a kind of third-order delay differential equations." Applications and Applied Mathematics: An International Journal, vol. 10, no. 2, pp. 772-782, 2015.

[21] M. Remili and L. Oudjedi, "Stability and boundedness of the solutions of nonautonomous thirdorder differential equations with delay." Acta Univ. Palacki. Olomuc., Far. rer. nat., Mathematica, vol. 35, no. 2, pp. 139-147, 2014.

[22] A. Rontó, M. Rontó, and I. Varga, "Partially solved differential system with two point nonlinear boundary conditions." Miskolc Mathematical Notes, vol. 18, no. 2, pp. 1001-1014, 2017, doi: 10.18514/MMN.2017.2491.

[23] A. I. Sadek, "On the stability of solutions of some non-autonomous delay differential equations of the third-order." Asymptotic Anal., vol. 43, pp. 1-7, 2005.

[24] P. Shekhar, V. Dharmaiah, and G. Mahadevi, "Stability and boundedness of solutions of delay differential equations of third-order." IOSR J. Math., vol. 5, pp. 9-13, 2013.

[25] C. Tunç, "On the stability of solutions for non-autonomous delay differential equations of thirdorder." Iran J. Sci. Technol. A, vol. 32, pp. 261-273, 2008.

[26] C. Tunç, "On the stability and boundedness of solutions to third-order nonlinear differential equations with retarded argument." Nonlinear Dynam., vol. 57, no. 1-2, pp. 97-106, 2009, doi: 10.1007/s11071-008-9423-6.

[27] C. Tunç, "Bound of solutions to third-order nonlinear differential equations with bounded delay." J. Franklin Ins., vol. 347, no. 2, pp. 415-425, 2010, doi: 10.1016/j.jfranklin.2009.05.001.

[28] C. Tunç, "Stability and bounded of solutions to non-autonomous delay differential equations of third-order." Nonlinear Dynam., vol. 62, no. 4, pp. 945-953, 2010, doi: 10.1007/s11071-0109776-5.

[29] C. Tunç, "On the stability and boundedness of certain third-order nonautonomous differential equations of retarded type." Proyecciones, vol. 34, no. 2, pp. 147-159, 2015, doi: 10.4067/S071609172015000200004.

[30] R. Wu and X. Mao, "Existence and uniqueness of solutions of stochastic differential equations." Stochastics, vol. 11, pp. 19-32, 1983. 
Authors' addresses

\section{Ayman M. Mahmoud}

Ayman M. Mahmoud, Department of Mathematics, Faculty of Science, New Valley University, ElKhargah 72511, Egypt.

E-mail address: math_ayman27@yahoo.com

\section{Cemil Tunç}

Cemil Tunç, Department of Mathematics, Faculty of Science, Yuzuncu Yil University, 65080, VanTurkey.

E-mail address: cemtunc@yahoo.com 